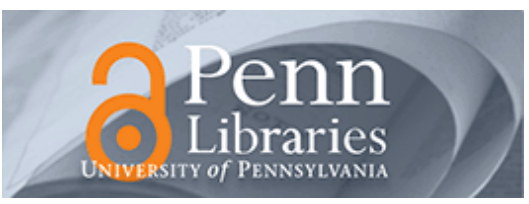

University of Pennsylvania

ScholarlyCommons

\title{
$2-24-2011$
}

\section{Electromagnetic tunneling through a single-negative slab paired with a double-positive bilayer}

\author{
Giuseppe Castaldi \\ University of Sannio \\ Ilaria Gallina \\ University of Sannio \\ Vincenzo Galdi \\ University of Sannio \\ Andrea Alù \\ University of Texas at Austin \\ Nadar Engheta \\ University of Pennsylvania \\ Follow this and additional works at: https://repository.upenn.edu/ese_papers \\ Part of the Electrical and Computer Engineering Commons
}

\section{Recommended Citation}

Giuseppe Castaldi, Ilaria Gallina, Vincenzo Galdi, Andrea Alù, and Nadar Engheta, "Electromagnetic tunneling through a single-negative slab paired with a double-positive bilayer", . February 2011.

Suggested Citation:

Reprinted from Physical Review B, Vol. 83, 081105(R).

Copyright 2011 American Institute of Physics. This article may be downloaded for personal use only. Any other use requires prior permission of the author and the American Institute of Physics.

The following article appeared in Physical Review B and may be found at http://dx.doi.org/10.1103/

PhysRevB.83.081105

This paper is posted at ScholarlyCommons. https://repository.upenn.edu/ese_papers/595

For more information, please contact repository@pobox.upenn.edu. 


\title{
Electromagnetic tunneling through a single-negative slab paired with a double- positive bilayer
}

\author{
Abstract \\ We show that resonant tunneling of electromagnetic fields can occur through a three-layer structure \\ composed of a single-negative (i.e., either negative permittivity or negative permeability) slab paired with \\ a bilayer made of double-positive (i.e., positive permittivity and permeability) media. In particular, one of \\ the two double-positive media can be chosen arbitrarily (even vacuum), while the other may exhibit \\ extreme (either near-zero or very high) permittivity and permeability values. Our results on this \\ counterintuitive tunneling phenomenon also demonstrate the possibility of synthesizing double-positive \\ slabs that effectively exhibit single-negative-like wave-impedance properties within a moderately wide \\ frequency range. \\ Disciplines \\ Electrical and Computer Engineering | Engineering

\section{Comments} \\ Suggested Citation: \\ Reprinted from Physical Review B, Vol. 83, 081105(R). \\ Copyright 2011 American Institute of Physics. This article may be downloaded for personal use only. Any \\ other use requires prior permission of the author and the American Institute of Physics. \\ The following article appeared in Physical Review B and may be found at http://dx.doi.org/10.1103/ \\ PhysRevB.83.081105
}




\title{
Electromagnetic tunneling through a single-negative slab paired with a double-positive bilayer
}

\author{
Giuseppe Castaldi, ${ }^{1}$ Ilaria Gallina, ${ }^{1}$ Vincenzo Galdi, ${ }^{1,}$ ' Andrea Alù, ${ }^{2}$ and Nader Engheta ${ }^{3}$ \\ ${ }^{1}$ Waves Group, Department of Engineering, University of Sannio, I-82100 Benevento, Italy \\ ${ }^{2}$ Department of Electrical and Computer Engineering, The University of Texas at Austin, Austin, Texas 78712, USA \\ ${ }^{3}$ Department of Electrical and Systems Engineering, University of Pennsylvania, Philadelphia, Pennsylvania 19104, USA
}

(Received 23 December 2010; published 24 February 2011)

\begin{abstract}
We show that resonant tunneling of electromagnetic fields can occur through a three-layer structure composed of a single-negative (i.e., either negative permittivity or negative permeability) slab paired with a bilayer made of double-positive (i.e., positive permittivity and permeability) media. In particular, one of the two double-positive media can be chosen arbitrarily (even vacuum), while the other may exhibit extreme (either near-zero or very high) permittivity and permeability values. Our results on this counterintuitive tunneling phenomenon also demonstrate the possibility of synthesizing double-positive slabs that effectively exhibit single-negative-like wave-impedance properties within a moderately wide frequency range.
\end{abstract}

DOI: 10.1103/PhysRevB.83.081105

PACS number(s): 42.25.Bs, 41.20.Jb, 78.20.Ci

The past decade has witnessed a growing interest in the study and applications of extraordinary transmission of electromagnetic (EM) fields through opaque media such as metals or, more general, single-negative (SNG) materials characterized by negative permittivity or permeability, and hence evanescent fields. With reference to metallic films, surface-plasmon-induced transparency was demonstrated theoretically and experimentally in Ref. 1, and a large body of results are available in connection with extraordinary transmission through subwavelength apertures (see, e.g., Ref. 2 for a recent review). In connection with general SNG media, it was shown in Ref. 3 that, in spite of their inherent opacity, slabs of (homogeneous, isotropic) epsilon-negative (ENG) and mu-negative (MNG) media can give rise to resonant tunneling phenomena (with total transmission and zero phase delay) when paired as a bilayer. Starting from this basic configuration, several extensions and generalizations have been investigated (see, e.g., Refs. 4-11).

Moreover, multilayer metallodielectric structures have been extensively studied in connection with near-field subwavelength imaging (see, e.g., Refs. 12-15), generating a growing interest in the study of resonant tunneling of evanescent waves in configurations featuring SNG media paired with double-positive (DPS) (i.e., positive permittivty and permeability) materials. Interestingly, while it is impossible to achieve perfect transparency by pairing a single SNG slab with an arbitrary DPS one, anomalous tunneling phenomena have been demonstrated in configurations featuring an SNG slab sandwiched between suitable impedance-matching DPS layers. ${ }^{16,17}$ In particular, the results in Ref. 16, pertaining to a symmetrical DPS-ENG-DPS three layer and experimentally validated at microwave frequencies, have been extended in Ref. 18 to more general (e.g., optical, quantum-mechanical) tunnel barriers. Against the above background, we propose here another counterintuitive resonant tunneling mechanism, which entails pairing an SNG slab with a bilayer made of DPS media. Unlike the configuration in Ref. 16, where the SNG slab is symmetrically sandwiched between two identical DPS layers, in our case the DPS layers are different and paired at one side only. This also allows direct comparisons with configurations involving paired ENG-MNG media, with the DPS bilayer playing the role of an effective SNG slab.
The problem geometry is illustrated in the Cartesian $(x, y, z)$ coordinate system of Fig. 1. Without loss of generality, we consider a homogeneous, isotropic ENG slab with relative permittivity $\varepsilon_{1}<0$ and thickness $d_{1}$ paired with a bilayer composed of homogeneous, isotropic DPS media with parameters $\varepsilon_{2}>0, d_{2}$ and $\varepsilon_{3}>0, d_{3}$, respectively, all embedded in vacuum $(\varepsilon=1)$ and assumed as nonmagnetic (i.e., $\mu=1)$ under time-harmonic $[\exp (-i \omega t)]$ plane-wave illumination.

Assuming normal incidence, for the ideal case of lossless media, we can derive via straightforward transfer-matrix algebra $^{19}$ the general resonance condition for total transmission by zeroing the reflection coefficient, viz.,

$$
\begin{aligned}
& i\left(1-\varepsilon_{1}\right) \sqrt{\varepsilon_{2} \varepsilon_{3}} \tau_{1}+i\left(1-\varepsilon_{2}\right) \sqrt{-\varepsilon_{1} \varepsilon_{3}} \tau_{2} \\
& \quad+i\left(1-\varepsilon_{3}\right) \sqrt{-\varepsilon_{1} \varepsilon_{2}} \tau_{3}+\sqrt{\varepsilon_{3}}\left(\varepsilon_{2}-\varepsilon_{1}\right) \tau_{1} \tau_{2} \\
& \quad+\sqrt{\varepsilon_{2}}\left(\varepsilon_{3}-\varepsilon_{1}\right) \tau_{1} \tau_{3}+\sqrt{-\varepsilon_{1}}\left(\varepsilon_{3}-\varepsilon_{2}\right) \tau_{2} \tau_{3} \\
& \quad+i\left(\varepsilon_{1} \varepsilon_{3}-\varepsilon_{2}\right) \tau_{1} \tau_{2} \tau_{3}=0,
\end{aligned}
$$

where $\tau_{1}=\tanh \left(k \sqrt{-\varepsilon_{1}} d_{1}\right)$ and $\tau_{2,3}=\tan \left(k \sqrt{\varepsilon_{2,3}} d_{2,3}\right)$, with $k=\omega / c=2 \pi / \lambda$ denoting the vacuum wave number, and $c$ and $\lambda$ denoting the corresponding wave speed and wavelength, respectively. Zeroing the real part of (1), we obtain

$$
\tau_{2}=\frac{\sqrt{\varepsilon_{2}}\left(\varepsilon_{1}-\varepsilon_{3}\right) \tau_{1} \tau_{3}}{\sqrt{\varepsilon_{3}}\left(\varepsilon_{2}-\varepsilon_{1}\right) \tau_{1}+\sqrt{-\varepsilon_{1}}\left(\varepsilon_{3}-\varepsilon_{2}\right) \tau_{3}},
$$

which, enforced in the imaginary part of (1), yields

$$
\tau_{3}= \pm \sqrt{\frac{\varepsilon_{3}\left(1-\varepsilon_{1}\right)\left(\varepsilon_{2}-\varepsilon_{1}\right) \tau_{1}^{2}}{\varepsilon_{1}\left(1-\varepsilon_{3}\right)\left(\varepsilon_{3}-\varepsilon_{2}\right)+\left(\varepsilon_{3}-\varepsilon_{1}\right)\left(\varepsilon_{3} \varepsilon_{1}-\varepsilon_{2}\right) \tau_{1}^{2}}} .
$$

Noting that the numerator of the square-root argument in (3) is always positive (since $\varepsilon_{1}<0$ and $\varepsilon_{2,3}>0$ ), real solutions exist if

$$
\varepsilon_{1}\left(1-\varepsilon_{3}\right)\left(\varepsilon_{3}-\varepsilon_{2}\right)+\left(\varepsilon_{3}-\varepsilon_{1}\right)\left(\varepsilon_{3} \varepsilon_{1}-\varepsilon_{2}\right) \tau_{1}^{2}>0 .
$$

The above inequality is quadratic in $\varepsilon_{3}$ (with positive discriminant), and yields the conditions

$$
\varepsilon_{3} \leqslant \varepsilon_{3 a} \text { or } \varepsilon_{3} \geqslant \varepsilon_{3 b},
$$

with $\varepsilon_{3 a}$ and $\varepsilon_{3 b}$ denoting the two (positive) roots of the denominator of the square-root argument in (3). It can be 


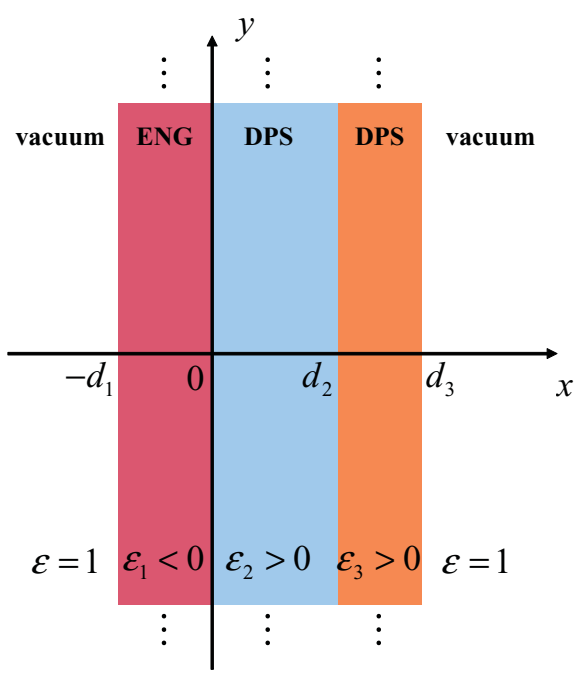

FIG. 1. (Color online) Problem geometry (details in the text).

readily shown from (4) that $\varepsilon_{3 a}<\varepsilon_{2}$ and $\varepsilon_{3 b}=\varepsilon_{2} / \varepsilon_{3 a}>\varepsilon_{2}$, from which emerges the impossibility of achieving total transmission by pairing an ENG slab with a single DPS slab in vacuum, consistent with Ref. 16. Conversely, in the presence of a DPS bilayer for a given ENG slab with parameters $\varepsilon_{1}, d_{1}$, it is always possible [from (2)-(5)] to find suitable parameters for the DPS layers so as to fulfill the total-transmission condition in (1) at a given frequency. More specifically, the parameter $\epsilon_{2}$ turns out to be completely arbitrary (but positive), while $\varepsilon_{3}$, $d_{3}$, and $d_{2}$ are derived proceeding backward from (5), (3), and (2), respectively.

A few general considerations are in order. First, at variance with the symmetrical DPS-ENG-DPS configuration in Ref. 16, no critical thickness value exists for our asymmetrical ENGDPS-DPS configuration in order to exhibit EM tunneling. In fact, there always exist four distinct classes of solutions for total transmission, corresponding to the possible combinations of the choices in (3) and (5). We found that, in the longwavelength limit $d_{n} / \lambda_{0} \rightarrow 0, n=1,2,3$, all these solutions tend toward the effective-medium-theory prediction obtained from (1) by neglecting the multiple-scattering terms (i.e., those containing multiple $\tau$ terms)

$$
\varepsilon_{1} d_{1}+\varepsilon_{2} d_{2}+\varepsilon_{3} d_{3}=d_{1}+d_{2}+d_{3} .
$$

It can be shown that, for an increasing opacity of the ENG medium $\left(\left|\varepsilon_{1}\right| \gg 1\right)$, the end values in (5) tend to exhibit extreme values, so the outermost DPS slab must have either near-zero $\left(\varepsilon_{3 a} \ll 1\right)$ or very-high $\left(\varepsilon_{3 b} \gg 1\right)$ permittivity. From (3), it is readily verified that the solutions associated to the end values $\varepsilon_{3}=\varepsilon_{3 a}$ or $\varepsilon_{3}=\varepsilon_{3 b}$ in (5) correspond to a quarterwavelength (plus half-wavelength periodicities) size for the outermost DPS slab, which (recalling its wave-impedance transformation properties ${ }^{19}$ ) is also representative of a different configuration featuring an ENG-DPS bilayer terminated with a DPS half-space (i.e., $d_{3} \rightarrow \infty$ ). Moreover, we highlight that the complete arbitrariness in the choice of $\varepsilon_{2}$ represents an important degree of freedom in the proposed configuration. In particular, choosing $\varepsilon_{2}=1$, we obtain another interesting tunneling configuration featuring an ENG slab paired with a
DPS slab via a separating vacuum layer. Finally, it can be shown that enforcing a purely real (i.e., \pm 1 ) transmission coefficient results in

$$
\begin{aligned}
& \left(\varepsilon_{1}^{2}-\varepsilon_{1} \varepsilon_{2}-\varepsilon_{1} \varepsilon_{3}+\varepsilon_{2} \varepsilon_{3}\right)^{2} \\
& =\left(1-\varepsilon_{1}\right)\left(\varepsilon_{2}-\varepsilon_{1}\right)\left[\frac{\varepsilon_{1}}{\tau_{1}^{2}}\left(\varepsilon_{3}-\varepsilon_{2}\right)\left(\varepsilon_{3}-1\right)\right. \\
& \left.\quad+\left(\varepsilon_{1}-\varepsilon_{3}\right)\left(\varepsilon_{1} \varepsilon_{3}-\varepsilon_{2}\right)\right]
\end{aligned}
$$

i.e., a quadratic equation in $\varepsilon_{3}$, the solutions of which, enforced in (4), yield the constraint

$$
\varepsilon_{1}^{2}\left(1-\varepsilon_{1}\right)\left(\varepsilon_{1}-\varepsilon_{2}\right)^{3} \tau_{1}^{2}>0,
$$

which is clearly impossible to fulfill with $\varepsilon_{1}<0$ and $\varepsilon_{2}>0$, thereby implying that no tunneling with zero phase delay is possible in our proposed configuration.

As a first example, we consider an ideal lossless configuration featuring an ENG slab with $\varepsilon_{1}=-3$ and $d_{1}=0.1 \lambda_{0}$ (here and henceforth, the subscript 0 identifies resonant frequency and wavelength quantities). We arbitrarily select $\varepsilon_{2}=2.5$, and readily derive from (2)-(5) the remaining parameters: $\varepsilon_{3}=$ $\varepsilon_{3 b}=16, d_{3}=0.0624 \lambda_{0}$ [choosing the positive determination for $\tau_{3}$ in (3)], and $d_{2}=0.236 \lambda_{0}$. For a normally incident (along the positive $x$ direction) plane-wave excitation, Fig. 2 illustrates the resonant electric and magnetic field (normalized) longitudinal distributions, from which can be observed the total-transmission effect, achieved via a growing evanescent wave in the ENG layer and a standing wave in the DPS bilayer. The electric field peaks toward the center of the middle DPS layer, and the magnetic field peaks at its boundaries, similar to a Fabry-Perot etalon, although we consider here the presence of an inherently opaque material. It is insightful to compare the above phenomenon with the tunneling occurring in an ENG-MNG pair. From the ENG-MNG matching conditions [cf. Eq. (8) in Ref. 3], we can straightforwardly derive the constitutive parameters $\varepsilon_{e}=1$ and $\mu_{e}=-0.33$ of the required $\mathrm{MNG}$ slab of same thickness $\left(d_{2}+d_{3}\right)$ as the above

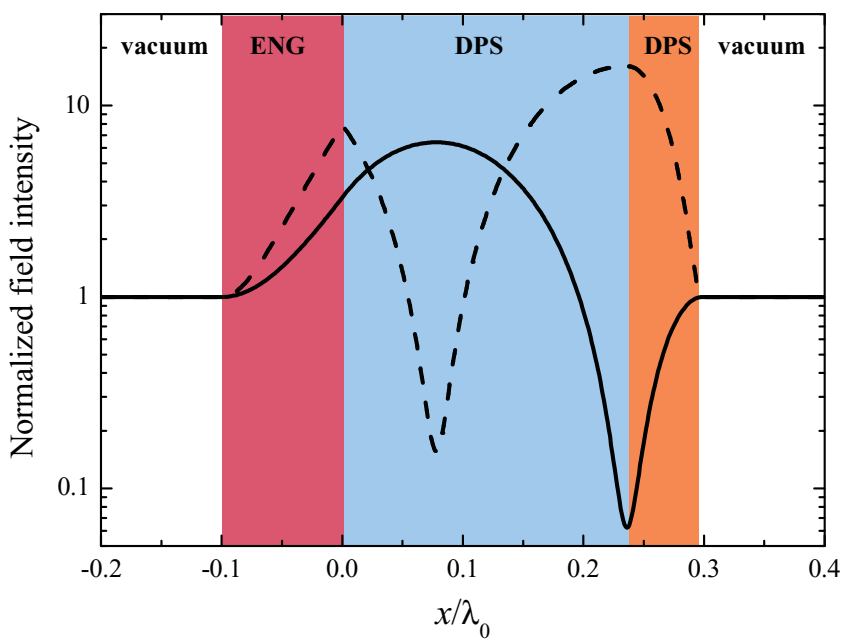

FIG. 2. (Color online) Resonant electric (solid) and magnetic (dashed) field intensity distributions (normalized by the incident fields) for a configuration as in Fig. 1 , with $\varepsilon_{1}=-3, d_{1}=0.1 \lambda_{0}$, $\varepsilon_{2}=2.5, d_{2}=0.236 \lambda_{0}, \varepsilon_{3}=16$, and $d_{3}=0.0624 \lambda_{0}$. 



FIG. 3. (Color online) (a) Reflection coefficient magnitude and (b) phase of the isolated DPS bilayer in Fig. 2 (blue solid line) as a function of the frequency, compared with the response (red dashed line) of an effective MNG slab with $\varepsilon_{e}=1, \mu_{e}=-0.33$.

DPS bilayer to "compensate" the given ENG slab. Figure 3 compares the reflection-coefficient responses of the isolated DPS bilayer and such MNG slab (free standing in vacuum) as a function of frequency. As expected, both responses result in large reflectivity (due to the opacity of an MNG standalone layer) and perfectly match at the resonance frequency. In particular, the designed DPS bilayer is capable of providing the capacitive input impedance required to resonate with the ENG slab, ensuring total transmission. The reflection coefficients in Fig. 3 agree reasonably well within a moderate bandwidth (maximum difference of $\sim 0.5 \%$ in magnitude and $\sim \pi / 12$ in phase, over a $10 \%$ bandwidth), ensuring that the designed DPS bilayer may effectively replace an MNG layer for a variety of applications.

Next, we move on to assessing the frequency and angular dependence, as well as the sensitivity to polarization and losses, of the above tunneling phenomenon. Figures 4 and 5 show the transmittance as a function of frequency and incidence angle (from the $x$ axis, for both $P$ and $S$ polarizations), respectively, for three representative parameter configurations. We start from the same configuration in Fig. 2, but now assuming a more realistic Drude-type dispersive, lossy model for the ENG medium,

$$
\varepsilon_{1}(\omega)=1-\frac{\omega_{p 1}^{2}}{\omega\left(\omega+i \gamma_{1}\right)},
$$

with the plasma angular frequency $\omega_{p 1}$ and the damping coefficient $\gamma_{1}$ chosen so as to ensure $\operatorname{Re}\left[\varepsilon_{1}\left(\omega_{0}\right)\right] \approx-3$ (with a loss tangent $\sim 10^{-2}$ ), and a nondispersive slightly lossy (loss tangent $=10^{-3}$ ) model for the DPS layers. From Fig. 4 (black solid curve), a rather broad resonance is observed in this case, with a peak transmittance of nearly $97 \%$, and a slow decay for higher frequencies attributable to the increasing transparency (approaching the plasma frequency) of the ENG slab. Also, the angular response (see Fig. 5) turns out not to be very selective, especially for $P$ polarization. As previously highlighted, tunneling effects may be obtained, in principle, for arbitrary choices of the ENG slab parameters and the permittivity of the middle DPS layer. For an increased opacity

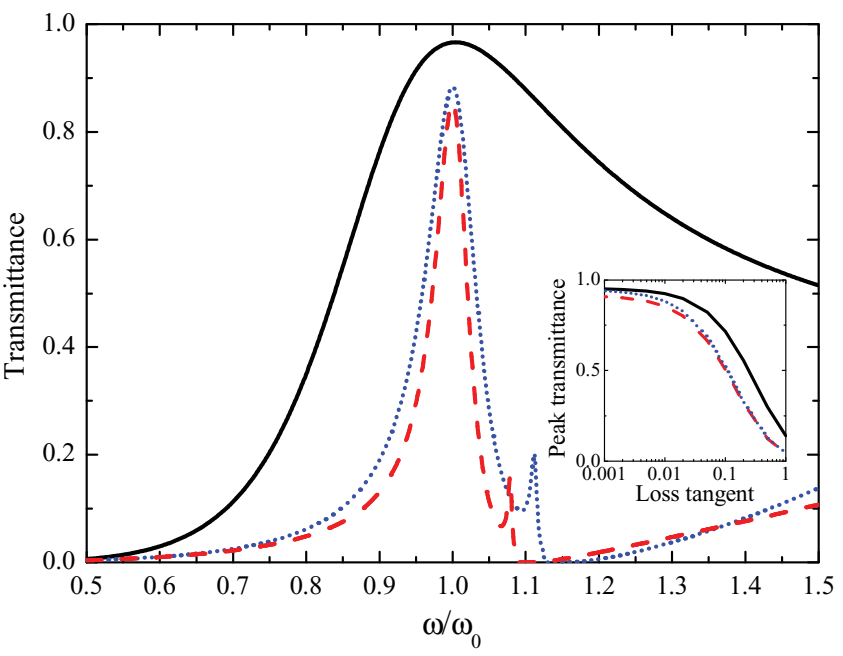

FIG. 4. (Color online) Black solid line: Transmittance as a function of the frequency for the parameter configuration in Fig. 2, but using for the ENG slab the dispersive model in (9) with $\omega_{p 1}=2 \omega_{0}, \gamma_{1}=3.75 \times 10^{-3} \omega_{p 1}$ \{i.e., $\left.\operatorname{Re}\left[\varepsilon_{3}\left(\omega_{0}\right)\right] \approx-3\right\}$, and a loss tangent of $10^{-3}$ for the DPS layers. Also shown are the responses obtained for an increased opacity of the ENG slab $\left\{\omega_{p 1}=10.05 \omega_{0}\right.$ and $\gamma_{1}=9.8 \times 10^{-4} \omega_{p 1}$ in (9), i.e., $\operatorname{Re}\left[\epsilon_{1}\left(\omega_{0}\right)\right] \approx-100$, and $d_{1}=$ $\left.0.01 \lambda_{0}\right\}$ for $\varepsilon_{2}=12$ (with loss tangent $=10^{-3}$ ) and $d_{2}=0.119 \lambda_{0}$ (red dashed line), and $\varepsilon_{2}=1$ and $d_{2}=0.472 \lambda_{0}$ (blue dotted line), with the high-permittivity outermost DPS layer described by the model in (10), with $\varepsilon_{3 \infty}=4, \Lambda_{3}=3.081 \omega_{3}$, and $\omega_{3}=1.091 \omega_{0}$, $\gamma_{3}=9.26 \times 10^{-4} \omega_{3}$ \{i.e., $\left.\operatorname{Re}\left[\varepsilon_{3}\left(\omega_{0}\right)\right]=63.7\right\}, d_{3}=0.036 \lambda_{0}$, and $\omega_{3}=1.13 \omega_{0}, \gamma_{3}=1.33 \times 10^{-3} \omega_{3}$ \{i.e., $\left.\operatorname{Re}\left[\varepsilon_{3}\left(\omega_{0}\right)\right]=47.8\right\}, d_{3}=$ $0.0313 \lambda_{0}$, respectively. The inset illustrates the influence of losses in the ENG material on the peak transmittance (as a function of the loss tangent at resonance).

of the ENG medium, as anticipated, the constitutive parameters of the outermost DPS layer tend to exhibit extreme values. For instance, considering an ENG slab with $\varepsilon_{1}=-100$ and $d_{1}=0.01 \lambda_{0}$, and $\varepsilon_{2}=12$, we obtain [choosing the larger end value $\varepsilon_{3 b}$ in (5)] $\varepsilon_{3}=63.7$. In Ref. 16, such (positive or negative) high-permittivity media were successfully synthesized at

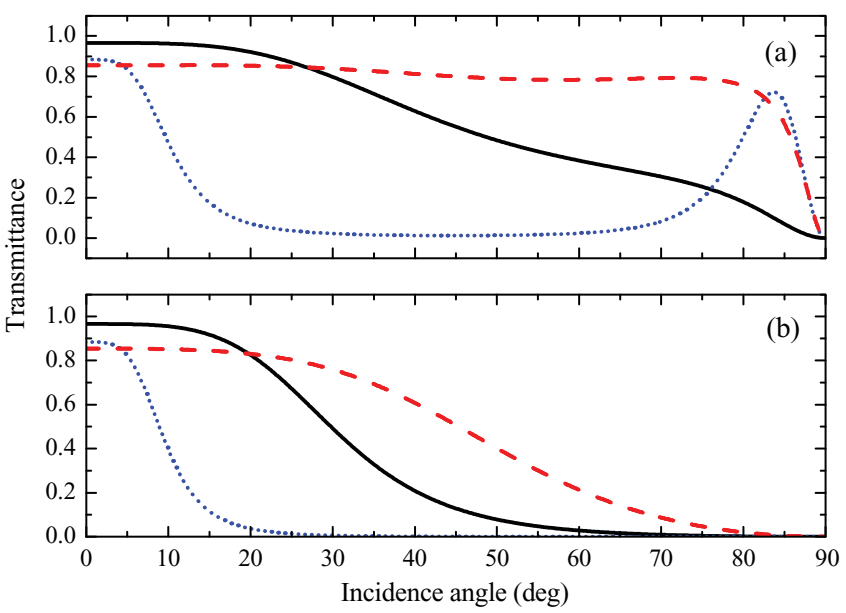

FIG. 5. (Color online) As in Fig. 4, but as a function of the incidence angle at the resonant frequency, for the (a) $P$ and (b) $S$ polarizations. 
microwave frequencies with resonant inclusions. Assuming for the outermost DPS medium a Lorentz-type dispersive, lossy model

$$
\varepsilon_{3}(\omega)=\varepsilon_{3 \infty}-\frac{\Lambda_{3}^{2}}{\omega^{2}-\omega_{3}^{2}+2 i \gamma_{3} \omega},
$$

with the parameters (given in Fig. 4 caption) tuned so as to ensure the required real part at the given frequency, we observe in Fig. 4 (red dashed curve) a narrower bandwidth (with peak transmittance of nearly $85 \%$ due to the larger sensitivity to losses, and a smaller transmission peak attributable to dispersion effects), and from Fig. 5 a flatter angular response (especially for $P$ polarization), which is a direct consequence of the increased permittivity values. ${ }^{16}$ The resonant field distributions, not shown here for brevity, are qualitatively similar to those in Fig. 2, with intensity enhancements of nearly a factor of 60. Also shown in Figs. 4 and 5 (blue dotted curves) are the responses obtained for the same ENG slab, but choosing $\varepsilon_{2}=1$, i.e., an ENG-vacuum-DPS configuration. While the frequency response and the field distributions (again, not shown for brevity) resemble the previous example, the angular response is now much more selective (for both polarizations) and exhibits (in the $P$ polarization case) another peak for neargrazing incidence, which corresponds to a pseudo-Brewster angle of the ENG slab. The sensitivity to losses in the ENG material is illustrated in the inset of Fig. 4 in terms of the peak transmittance as a function of the loss tangent (at resonance), and qualitatively resembles the behavior observed in other tunneling phenomena involving SNG materials. ${ }^{3}$ Finally, our simulations show that, for given parameters of the ENG slab, the sensitivity to variations within $\sim 1 / 25$ of the local wavelength with respect to the nominal DPS slab thicknesses $d_{2,3}$ would still yield moderate reductions $(\sim-3 \mathrm{~dB})$ of the peak transmittance (with possible frequency shifts).

To sum up, we have illustrated a counterintuitive EM-field resonant tunneling mechanism that can take place by pairing an SNG slab with a DPS bilayer. In particular, we have worked out analytically the design criteria and explored the dependence on frequency, incidence direction, polarization, and material losses. Our results, which can be extended (along the lines of Refs. 18 and 20) to more general (e.g., optical, quantum-mechanical) asymmetrical tunnel barriers, also allow direct comparisons with ENG-MNG paired configurations, and can be understood in terms of the equivalent waveimpedance properties exhibited by a DPS bilayer and a "matched" (according to Ref. 3) SNG slab. This observation may suggest more general application scenarios of such a DPS bilayer, for which SNG-like responses may be emulated via simpler dielectric slabs, which may somehow compensate, in a simple geometry, opaque ENG metamaterial slabs for a variety of applications in which complementary metamaterials are paired together. ${ }^{3,4}$ Also of interest is the possible adaptation for applications to super-resolution imaging schemes. *vgaldi@unisannio.it

${ }^{1}$ R. Dragila, B. Luther-Davies, and S. Vukovic, Phys. Rev. Lett. 55, 1117 (1985).

${ }^{2}$ F. J. García-Vidal, L. Martín-Moreno, T. W. Ebbesen, and L. Kuipers, Rev. Mod. Phys. 82, 729 (2010).

${ }^{3}$ A. Alù and N. Engheta, IEEE Trans. Antennas Propag. 51, 2558 (2003).

${ }^{4}$ J. B. Pendry and S. A. Ramakrishna, J. Phys. Condens. Matter 15, 6345 (2003).

${ }^{5}$ G. Castaldi, I. Gallina, V. Galdi, A. Alù, and N. Engheta, J. Opt. (Issy-les-Moulineaux, Fr.) 13, 024011 (2011).

${ }^{6}$ T. Feng, Y. Li, H. Jiang, Y. Sun, L. He, H. Li, Y. Zhang, Y. Shi, and H. Chen, Phys. Rev. E 79, 026601 (2009).

${ }^{7}$ Y. Ding, Y. Li, H. Jiang, and H. Chen, PIERS Online 6, 109 (2010).

${ }^{8}$ H. Jiang, H. Chen, H. Li, Y. Zhang, J. Zi, and S. Zhu, Phys. Rev. E 69, 066607 (2004).

${ }^{9}$ G. Guan, H. Jiang, H. Li, Y. Zhang, H. Chen, and S. Zhu, Appl. Phys. Lett. 88, 211112 (2006).
${ }^{10} \mathrm{X}$. Zhou and G. Hu, J. Opt. A: Pure Appl. Opt. 9, 60 (2007).

${ }^{11}$ Y. Fang and S. He, Phys. Rev. A 78, 023813 (2008).

${ }^{12}$ S. A. Ramakrishna and J. B. Pendry, Phys. Rev. B 67, 201101(R) (2003).

${ }^{13}$ W. Cai, D. A. Genov, and V. M. Shalaev, Phys. Rev. B 72, 193101 (2005).

${ }^{14}$ P. A. Belov and Y. Hao, Phys. Rev. B 73, 113110 (2006).

${ }^{15}$ B. Wood, J. B. Pendry, and D. P. Tsai, Phys. Rev. B 74, 115116 (2006).

${ }^{16}$ L. Zhou, W. Wen, C. T. Chan, and P. Sheng, Phys. Rev. Lett. 94, 243905 (2005)

${ }^{17}$ K.-Y. Kim and B. Lee, Phys. Rev. A 77, 023822 (2008).

${ }^{18}$ I. R. Hooper, T. W. Preist, and J. R. Sambles, Phys. Rev. Lett. 97, 053902 (2006).

${ }^{19}$ M. Born and E. Wolf, Principles of Optics, 7th ed. (Cambridge University Press, Cambridge, UK, 1999).

${ }^{20}$ L. Jelinek, J. D. Baena, J. Voves, and R. Marques, e-print arXiv:1011.4649v1. 